\title{
Development of HEVNP as a Multifunctional Strategy to Combat SARS-CoV2 as Preventive Vaccine and Antiviral Drug
}

\author{
Chun-Chieh Chen ${ }^{1,2,3}$, Mo A Baikoghli ${ }^{1,4}$ and R Holland Cheng ${ }^{1 *}$ \\ ${ }^{1}$ Department of Molecular and Cellular Biology, University of California, Davis, CA, USA \\ ${ }^{2}$ Department of Dermatology, University of California, Sacramento, CA, USA \\ ${ }^{3}$ Astrid Pharma Corp., Davis, CA, USA \\ ${ }^{4}$ Institute for Molecular Medicine Finland (FIMM), HiLIFE, University of Helsinki, \\ Helsinki, Finland
}

*Corresponding Author: R Holland Cheng, Professor, Department of Molecular and

Cellular Biology, University of California, Davis, CA, USA.
Received: October 06, 2020

Published: November 30, 2020

(C) All rights are reserved by $\mathbf{R}$ Holland

Cheng., et al.

\begin{abstract}
Hepatitis E virus nanoparticle (HEVNP) is an orally stable, mucosa-penetrating delivery platform for noninvasive, targeted delivery of therapeutic and diagnostic agents. HEVNP does not carry HEV genomic RNA and is incapable of replication. The key characteristics that make HEVNP an ideal and unique vehicle for diagnostic and therapeutic delivery include surface plasticity, resistance to the harsh environment of the gastrointestinal (GI) tract, significant payload capacity, platform sustainability, and safety. Furthermore, HEVNP can be produced using currently established expression/purification technologies; it can be easily formulated as a liquid or powder and can be distributed (and stored) without the need for a temperature-controlled supply chain. Such a modularized platform proves effective in various applications, including cancer therapeutics, oral insulin delivery against diabetes, and the oral vaccine against infectious diseases. The capsid's flexibilities fulfill the desired function to conjugating different functional peptides and/or compounds onto the capsule surface and payloads into its interior. Here, we explore further applications of a modularized therapeutic vaccine against SARS-CoV2, including lung tissue targeting ligands displayed on the surface of HEVNP and encapsulation of DNA vectors co-expressing SARS-CoV2 antigens and shRNA to inhibit viral replication.
\end{abstract}

Keywords: SARS-CoV-2; Mucosa-Delivery; HepE Virus Nano Particle; Therapeutic Vaccine; RNAi

\section{Abbreviations}

SARS-CoV-2: Severe Acute Respiratory Syndrome Coronavirus 2; HEV: Hepatitis E Virus; HEVNP: Hepatitis E Virus Nanoparticle; VLP: Virus-Like Particle; RNAi: RNA Interference; shRNA: Short Hairpin RNA; Theranostic: Therapeutic and Diagnostic

\section{Introduction}

\section{Hepatitis E Virus Nanoparticles (HEVNP)}

Hepatitis E Virus Nanoparticle (HEVNP) is derived from a modified capsid of the hepatitis E virus (HEV), composing noninfectious, self-assembling capsids capable of cell-binding and entry.
HEVNP maintains its structural integrity in a low-pH environment [1], which is advantageous for oral administration and particularly for intratumoral penetration. HEVNP forms a hollow, icosahedral capsid composed of 60 identical units. Each subunit protein consists of three domains; shell, middle, and protrusion [3]. The accessible surface protrusion (P) domain connects through a flexible hinge to a stable icosahedral base. Based on our engineered design, the $\mathrm{P}$ domain is the preferable site for chemical conjugation that allows functional modification of HEVNP. With 60 repeated units of capsid proteins, single site-specific modification on the P domain results in anchoring sites to carry multivalent foreign molecules. Our results 
indicate that such engineering to the P domain occludes the native anti-HEV antibody recognition sites, which is highly beneficial for medical applications, discussed below [4].

We recently demonstrated that HEVNP-based theranostic capsule could target tissues of interest when conjugated with tumor-targeting peptides. Our engineered HEVNP conjugated with a breast cancer targeting ligand (LXY30) clearly showed its specific targeting to breast tumor cells both in vitro and in vivo. Thus, HEVNP can be manipulated to facilitate targeted delivery of diagnostic or therapeutic reagents to the tumor site [4]. Additionally, the use of HEVNP as an immunogen carrier has shown promising results. Recently, its display of HIV mimotopes successfully demonstrated the utilization of the HEVNP as a mucosa-penetrating delivery platform as oral vaccines [5]. A 15 residue peptide (P18) from the Env protein, HIV's surface glycoprotein, gp120, was genetically inserted next to residue Y485 of the truncated CP, a location within the antibody-binding site of HEVNP. Cryo-EM studies revealed that the HEVNP capsid maintained its icosahedral shape after the P18 insertion and upon administration in mice, resulted in a robust cytotoxic T cell and humoral response against HIV.

In addition to capsid functionalization, HEVNP can encapsulate within its hollow core he designated payload for delivery. It is a simple process that reversibly disassembles HEVNP and then reassembles it in the presence of payload molecules. We demonstrated that plasmid DNA, encoding HIV envelope gp120, can be orally administered in mice. This construct can orally deliver the plasmid to the spleen, Peyer's patches, and mesenteric lymph nodes of mice. Cell-mediated immune (specific cytotoxic T-lymphocyte (CTL) response) and specific humoral responses are generated locally and systemically by the DNA vaccine (Takamura., et al.). Thus, by combining its surface modification and interior encapsulation capabilities, HEVNP can function as a multifunctional therapeutic vaccine. HEVNP is a platform capable of mucosal delivery, and specific cell-targeting of peptide antigen and DNA expressed antigen to stimulate both B-cell and T-cell immune response (reviewed in [8,9]).

Translational approach for the use of HEVNP to address the challenges raised by COVID-19

Coronavirus disease 2019 (COVID-19) is an illness caused by a novel agent, severe acute respiratory syndrome coronavirus 2 (SARS-CoV-2). As of October of 2020, COVID-19 has claimed 1.2 million deaths and over 35 million cases worldwide. Coronaviruses are single-stranded positive-sense RNA viruses encapsulated within a membrane envelope. The viral membrane displays with a spike (S) glycoprotein that gives coronaviruses their crownlike appearance. There are four classes of coronaviruses designated as alpha, beta, gamma, and delta. The betacoronavirus class includes severe acute respiratory syndrome (SARS) virus (SARS-CoV), Middle East respiratory syndrome (MERS) virus (MERS-CoV), and the COVID-19 causative agent SARS-CoV-2 [10].

\section{$\mathrm{T}$ and $\mathrm{B}$ cell responses against SARS-CoV-2}

As indicated by prior research, the role of humoral and cellmediated immune responses in SARS-CoV (2003 pandemic), as summarized by Ahmed., et al. [14], can be a useful reference for SARS-CoV-2 vaccine design due to its high similarity. For the former SARS-CoV case, antibody responses generated against the spike (S) protein have shown protection against infection in mouse models [17-19]. Multiple studies have shown that antibodies generated against the $\mathrm{N}$ protein of SARS-CoV, a highly immunogenic and abundantly expressed protein during infection [20], were particularly prevalent in SARS-CoV-infected patients [21,22]. While being effective, the antibody response induced by $\mathrm{B}$ cells was found to be short-lived in convalescent SARS-CoV patients [23]. In contrast, $\mathrm{T}$ cell responses have been shown to provide long-term protection [23-25], even up to 11 years post-infection [26]. These have also attracted interest in a prospective vaccine against SARSCoV [reviewed in [27]].

Among the SARS-CoV component proteins, the structural proteins are the most immunogenic to respond to T cells in peripheral blood mononuclear cells of convalescent SARS-CoV patients compared to the nonstructural proteins [28]. Of the structural proteins, $\mathrm{T}$ cell responses against $\mathrm{S}$ and $\mathrm{N}$ proteins have reportedly the most dominant and long-lasting [29]. In SARS-CoV-2, both T and B cell responses are detected in the blood around one week after the onset of COVID-19 symptoms. CD8+ T cells are crucial for directly attacking and killing virus-infected cells, whereas CD4+ T cells are crucial to prime both CD8+ T cells and B cells. CD4+ T cells are also responsible for cytokine production to drive immune cell recruitment. Coronavirus-specific $\mathrm{T}$ cells are essential in eliminating the virus and controlling disease development and effective vaccine strategies [30]. However, whether T cell responses alone can prevent infection in human settings remains to be investigated. 
HEVNP for mucosal delivery of SARS-CoV2 vaccine and targeted RNAi

HEVNP is an ideal vehicle for mucosal delivery, driven by its structural rigidity of HEVNP and capacity to avoid degradation in low pH and highly enzymatic environments. The mucosal immune system is the first defense mechanism to several pathogens entering the body through the mucosal membranes. The mucosal immune activities are associated with mucosa-associated lymphoid tissues (MALT), present in mucosal tissue in the nose, lungs, gastrointestinal tract, and vaginal/rectal surfaces [36]. The MALT is classified into specific sub-compartments, depending on the location, including the gut-associated lymphoid tissue (GALT), nasopharynx-associated lymphoid tissue (NALT), bronchus-associated lymphoid tissue (BALT) [37]. The mucosal systems are protected by the immune cell that populates the region along the mucosal surfaces and epithelial cells and mucus that act as physical barriers before the pathogen gains access to the underlying tissue.

\section{RNA interfering (RNAi)}

RNA interference (RNAi), a primary antiviral defense mechanism in plants, can "silence" or suppress the expression of genes by small interfering RNA (siRNA). A class of doublestranded non-coding RNA molecules, 20 - 25 base pairs in length, is also known as short interfering RNA or silencing RNA. Based on the RNAi phenomenon, siRNA-based therapeutics have been developed and implemented for anticancer, antiviral, and genetic diseases [15]. Some earlier studies revealed that siRNA candidates were effectively used against SARS and Middle-East Respiratory Syndrome (MERS) [42]. In 2003, an siRNA-based drug was developed by Sirnaomics, Inc. (Maryland, USA) for the outbreak of SARS-CoV and H5N1 influenza. In 2006, specific sequences of the severe acute respiratory syndrome (SARS) viral RNA, targeted by small interference RNA (siRNA) or short hairpin RNA (shRNA), had proven effectively inhibiting viral RNA (vRNA) expression of the envelope, E, protein and viral RdRP [43].

The viral genome of SARS-CoV-2 is $29 \mathrm{kbp}$ in size and one of the largest genomes among the RNA virus. This genome consists of fourteen open reading frames (ORFs) coding for twentyseven structural and nonstructural proteins [44]. At the 5' end, there are the two largest ORFs, namely ORF1a and ORF1b, which are translated into a single large poly-protein by the ribosome through a frame-shift event. The ORF1a comprises of two viral cysteine proteases, namely papain-like protease (nsp3) and the main protease designated as 3-chymotrypsin-like protease or 3CL. Between the two viral proteases, the sequence coding for the nsp3 is reportedly to be less conserved [15]. However, the seq coding for the protease 3CL (nsp5) has been observed highly conserved among the annotated sequences [45]. Currently, the protease has been considered a primary drug target for multiple antiviral agents presently undergoing clinical trials. Thus, the sequence coding for nsp5 can treat as a potential target for RNAi using siRNA-based therapeutics.

Other potential targets include the viral RNA-dependent RNA polymerase (RdRP), located in the ORF1b, stretching from 13 - 16 $\mathrm{kb}$ on the viral genome. Downstream to the sequences from 16 $18 \mathrm{~kb}$ is the site coding for the viral helicase. These two sites are reportedly highly conserved among the annotated genome of SARSCoV-2 and the earlier genomes of beta coronaviruses like SARS and MERS [45]. Thus, these two sites can potentially the targets for RNAi using siRNA or shRNA.

\section{Experimental Design}

Our structure-guided approach aids the design of mucosal delivery in two folds:

1) HEVNP's protruding arms to functionalize with targeting or immunogenic peptides.

2) HEVNP's void interior to encapsulate heterologous nucleic acids.

The scientific premise is built upon decades of rational engineering based on HEVNP structure first established in the 90s and progressively refined by cryo-EM and X-ray crystallography [4648]. HEVNP's capsid arrangement poses a significant advantage in its modular surface that epitope engineering on its exterior does not alter its stability in an acidic and proteolytic environment [5].

Applying HEVNP as a mucosal vaccine (for instance, intranasal or oral) offers a novel solution, where this nanoplatform provides the capability to encapsulate therapeutic agents, such as SAg- DNA. HEVNP can simultaneously modulate the display of immunogenic peptides. We will take advantage of the naturally stable structure of the HEVNP for surface modulation $[4,5]$ and therapeutic cargo $[6,7,49]$.

Conjugation of functional peptides to the protruding arms of HEVNP enhances targeted delivery and mediates an escape from immune surveillance against HEV

A vital consideration for virus-based delivery systems is that the delivery systems themselves elicit immune responses, making the delivery system not reusable. We have found that the antigenicity of HEVNP lies exclusively within the surface P domain [50]. Thus, 
peptide conjugation of the $\mathrm{P}$ domain will eliminate or significantly reduce immune responses against HEVNP (or by pre-existing HEV antibodies) (Figure 1A) [4]. The effectiveness of epitope presentation on the surface of HEVNP is based on our extensive data that repeated use of HEVNP as a drug delivery system is not challenged by self- immunity. As we demonstrated, genetic insertion of a 15-residue V3-mimotope significantly lowered the immune HEV-surveillance since the genetic insertion occupied in the antibody-binding site after the residue Tyr485.

Further, the insertion of Env mimotope triggered an HIV-specific CTL response. Two antibodies tested the reactivity of p18-HEVNP, 447-52D, and HEP224, specifically probing HIV-V3 and HEV-capsid, respectively [5]. More recently, we applied cryo-EM to resolve the structure of HEVNP functionalized with mimotopes of Chlamydia muridarum at $2.8 \AA$ (data not shown). Two peptides derived from variable domains of $\mathrm{Cm}$, were chemically conjugated to the N573C position. The VD4-HEVNP induced a humoral response with a considerable amount of IgA production at mucosal surfaces. In combination with the $\mathrm{CpG}$ adjuvant, it triggered a robust cellmediated reaction [9].

\section{Encapsulation and delivery of heterologous nucleic acids}

Critical positively charged residues on the inner surface of the HEVNP allow encapsulation of negatively charged, nonsequencespecific DNA to be incorporated. Such assessment can be achieved by disassembling the particles into dimers using DTT and EGTA and subsequently allowing reassembly in the presence of the DNA in a calcium- or magnesium-rich environment. Thus, the HEVNP acts as a carrier for orally deliverable DNA vaccines (Figure 1B).
We encapsulated HIV-1 gag DNA plasmid into HEV VLPs and orally administered them in mice. Substantial humoral and cell-mediated immune responses were generated in local and systemic lymphoid organs. Notably, this oral DNA vaccine was successful in being delivered to lymphoid organs and allowed the particles to enter immune cells, undergo uncoating, and release the encapsulated DNA. Further, the gag gene expression took place successfully, where the Gag protein expressed in the host cells as a foreign antigen. The expressed antigen could be probed by immunoelectron microscopy in the gut epithelial sections, where Gagimmunogold was applied to investigate immunized mice $[8,49]$.

An intranasal deliverable therapeutic SARS-CoV-2 vaccine utilizing HEVNP will stimulate T-cell response and suppress viral RNA by RNA interference

To combat COVID 19, we utilize the modular property of the HEVNP as an intranasal delivery system for a combination of the ACE binding epitope for SARS-CoV-2 infected cell targeting, SAg DNA vaccine, and shRNA for the suppression of viral RNA silencing in SARS-CoV2 infected cells within the respiratory tract (Figure 2). The therapeutic vaccine, expressing shRNAs (in this case, it will target nonstructural proteins) in SARS-CoV-2 infected cells, can be used as nucleotide-based therapeutics to suppress the virus shedding by the infected patients and asymptomatic carriers. The major components of this therapeutic vaccine capsule are (1) SAg peptide epitope acting as not only a surface antigen to induce B cell immune response but also cell-specific ligand to target SARS-CoV2 infected cells; the dual DNA-expressed vector inserted with (2) SAg DNA as DNA vaccine to trigger T cell immune response, and (3) shRNA to suppress viral RNA titers in infected cells.
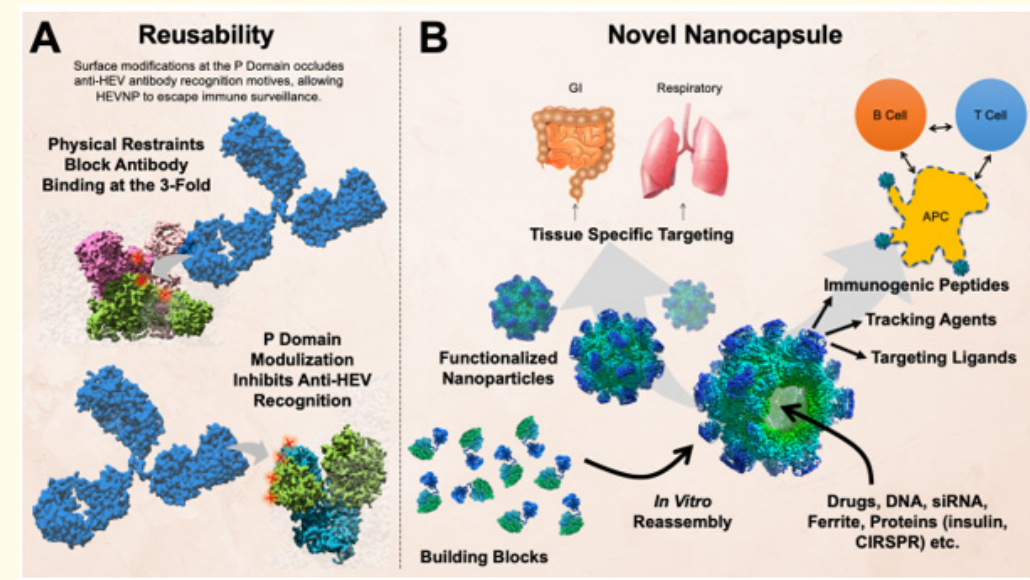

Figure 1: Use of HEVNP as an orally deliverable nanoparticle. (A) A major concern with the use of virus-like particles or virus-derived nanoparticles is the recognition of the immune system, impeding the reusability of the delivery system. HEVNP's anti-HEV antibody recognition sites are located on the P domain. When the P domain is modified, anti-HEV antibodies can no longer bind. Additionally, the 3-fold axis of HEVNP imposes additional constrictions for antibody binding by steric hindrance. (B) HEVNP is a novel Nanocapsule with capacity of tissue-targeted delivery of theranostics and vaccines. 


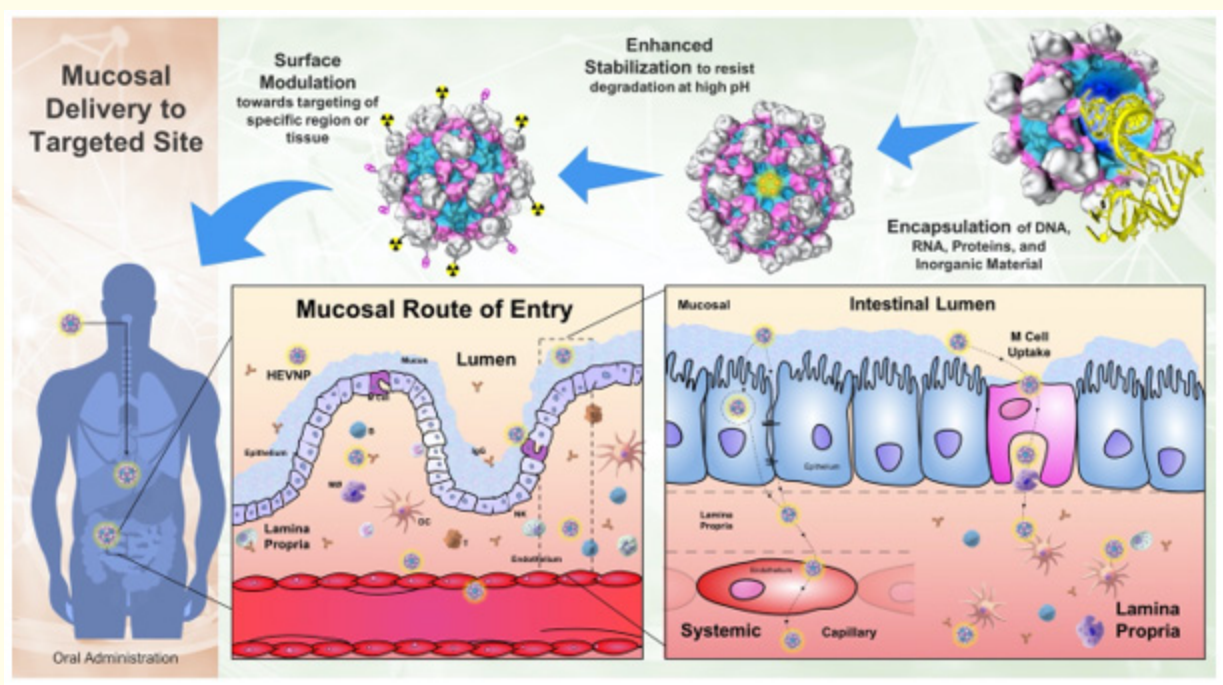

Figure 2: Mucosa-focused delivery. While HEVNP excludes the infectious genome of HEV, it retains the structural stability of the native virion; allowing it to penetrate the mucosal surfaces of the intestinal and nasopharynx tract. With the capacity of encapsulation of heterologous nucleic acid, proteins, and peptide-based therapeutics in addition to surface modulation to enhance penetration, retention, and targeting, the system can readily be used for mucosal delivery. In the context of vaccination, HEVNP can be transported from the lumen of the gut into the lamina propria (as well as through transcellular gaps) to reach the endothelium to achieve systemic delivery. Thus the system can act locally and systemically with enhanced targeting capacity.

The design of dual shRNA-antigen vector encapsulating into HEVNP with ACE2 targeting ligand.

Identifying specific crRNA sequences to target and cleave SARSCoV-2, bioinformatic analysis of aligning published SARS-CoV-2 genomes from 47 patients with SARS-CoV and MERS-CoV genomes were done by Abbott., et al. 2020 (Abbott., et al.). SARS-CoV-2 has a single-stranded RNA genome with $\sim 29,000$ nucleotides that encode 12 putative, functional open reading frames (McBride, van Zyl, and Fielding; JF Chan., et al.). We design the shRNAs with the most conservation between 47 SARS-CoV-2 strains, as well as SARS-CoV and MERS-CoV genomes. Two of the highly conserved regions include the RNA-dependent RNA polymerase (RdRP) gene in the polypeptide $\mathrm{ORF} 1 \mathrm{ab}$ region and the nucleocapsid $(\mathrm{N})$ gene at the end of the viral genome. Our shRNA candidates include:
1) 5'-UGAACCAAGACGCAGUAUUAUU-3', (N) gene
2) 5'-ACAGCAGCCAAACUAAUGGUUG-3', (RdRP) gene
3) 5'-ACAUAAUCAGGAUGUAAACUUA-3',(RdRP) gene
4) 5'-CAAACCCGGUAAUUUUAACAAA-3', (RdRP) gene.

The viral inhibition effect of each shRNA construct is analyzed using pseudo-virus (PV) constructs in the cell test described [57].
After the gene suppression effect is confirmed, the shRNA cassettes insert into antigens, such as spike (S) protein or RBD, expressed vectors in building dual shRNA-antigen vectors. The dual shRNAantigen vector will be encapsulated into HEVNPs following the described protocol as a potential antiviral vaccine against SARSCoV-2, influenza, and other coronaviruses due to their conserved RNA sequences and peptide sequences. To ensure that the dualfunction gene therapeutics are delivered to the SARS-CoV-2 infected tissues/cells with ACE highly expressed on the cell membrane, we add the RBD domain of spike protein as a targeting ligand on the surface of the delivery capsule, HEVNPs. Nonetheless, the RBD domain can act as an antigen to trigger the B cell immune system to benefit the therapeutic vaccine using HEVNPs.

\section{Expected Results and Discussion}

Prof. Holland Cheng's patent covering Hepatitis E Virus Nanoparticles (HEVNPs) (Cheng and Xing; Cheng., et al.), derived from a modified form of the HEV capsid protein, are non-infectious, self-assembling capsids capable of cell-binding and entry. Like the native virus, HEVNP is stable in an acidic environment and resistant to proteolytic digestion. Thus, it poses a great advantage as an oral delivery vehicle. Importantly, HEVNP can orally deliver plasmid 
DNA to the small intestine (epithelial cells) and induce antibody and cytotoxic T lymphocyte (CTL) responses against the plasmidencoded antigen. These studies have established the feasibility of utilizing HEVNP for mucosal delivery DNA vaccine carrier, in a route resembling virus native transmission.

HEVNP's unique attributes will be guiding our design to target mucosal surfaces and extreme tolerance to proteolytic degradation and acidic $\mathrm{pH}$, where other methods would fall short in terms of stability and retention capacity to reach the designated cells. Mucosal penetration is key to ensuring DNA vaccine success or RNP delivery into the infected and healthy cells. HEVNP is an ideal vehicle to carry gRNA and DNA vaccine against SARSCoV2 as it (i) possesses unique surface plasticity, (ii) is stable at room temperature, (iii) is really produced in large quantity, (iv) can readily package large biological molecules such as CRISPR complex, cas9/gRNA RNP, and condensed plasmid DNAs, (iv) can be delivered orally and intranasally as it is stable in low $\mathrm{pH}$; and (v) has the potential to directly target the accessible mucosa of the nasal cavity and the intestines (Figure 2 in the project?). Applying HEVNP as a mucosal vaccine (for instance, intranasal or oral) offers a novel solution, where this nanoplatform is engineered to have the capability of encapsulation of therapeutic agents, such as SAgDNA, to induce T cell immune response. The surface of HEVNP can be simultaneously modified to display immunogenic peptides to trigger B cell immune response. Taking advantage of RNAi, shRNA cassette, and the antigen genes, such as the Spike Protein (SP) gene of SARS-CoV-2, into plasmid vector as shRNA-antigen dual expressed DNA construct as a therapeutic vaccine. We re-purpose the use of our HEVNP to encapsulate dual shRNA-antigen DNA vector plasmid in its core, and with either chemical conjugating or genetic engineering ACE2-specific targeting ligands (derived from the RBD of SARS-CoV2 spike proteins) onto its surface to serve cell targeting (Summary figure 3).

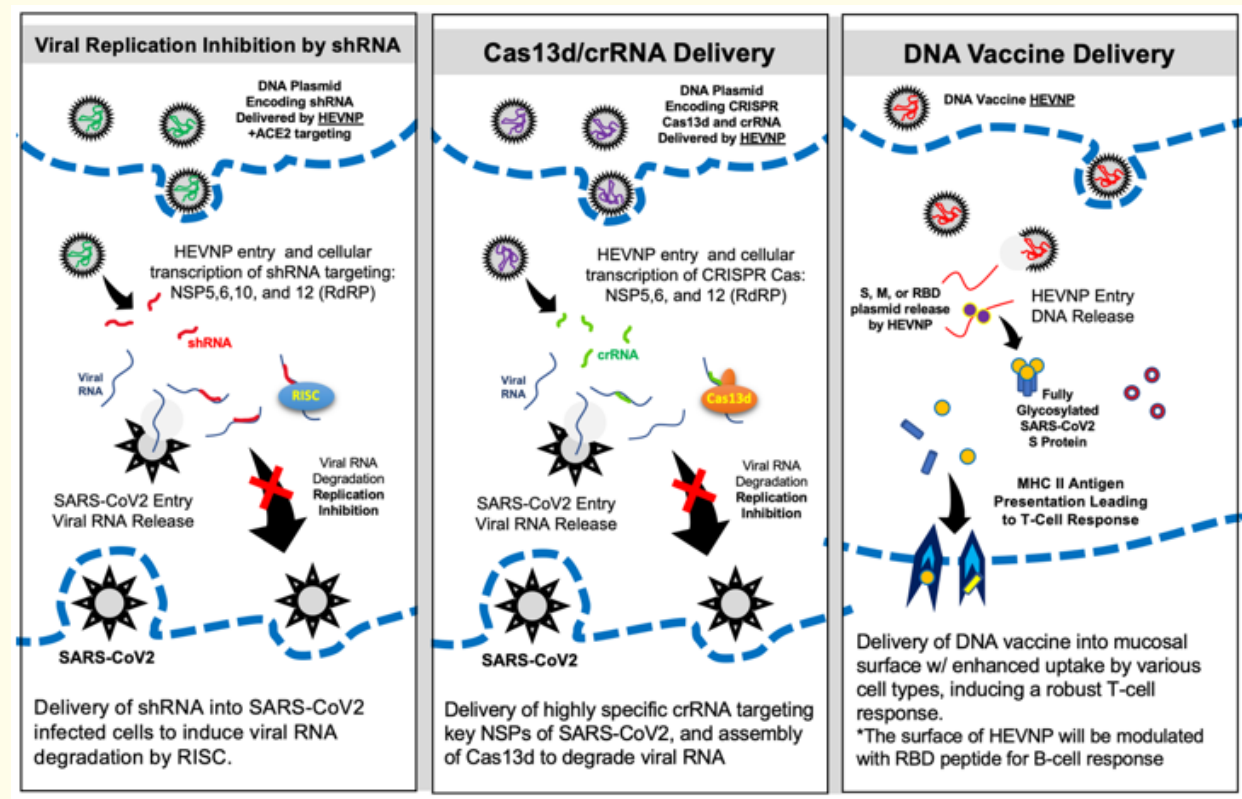

Figure 3: The use of HEVNP as a vaccine/therapeutics delivery against COVID-19. (A) Delivery of RNA interfering molecules such as shRNA can be achieved by encapsulating a DNA plasmid encoding antiviral RNA genome. Upon HEVNP's entry into the lung epithelia (enhanced by ACE2 targeting ligand; the primary receptor of SARS-CoV2), the DNA plasmid is released and translated into RNAi. shRNA can bind the viral RNA and facilitated by the RISC can result in viral RNA degradation. (B) Another approach can be the use of CRISPR Cas13d and CRISPR-associated RNA to target viral RNA and degrade viral RNA to inhibit SARS-CoV2 replication. (C) DNA vaccine can also be delivered into induce cell-mediated and humoral immune responses against SARS-CoV2. In this example, the S protein antigen, present on the surface of SARS-CoV2, can be delivered into lung epithelia to induce MHC-facilitated antigen presentation to T-cell; resulting in a robust immune response against SARS-CoV2. 


\section{Conclusion}

The use of HEVNP as our delivery vehicle offers several advantages: 1) highly efficient delivery of DNA to enter the nucleus, 2) highly adaptable DNA plasmid molecules encapsulation to allow rapid modification to probe the emerging challenges caused by viral gene mutations, and 3) minimized side-effects of nonspecific delivery. HEVNP stands out as a near-perfect platform for the needed responsiveness to the current outbreak with mucosal routes of deliveries to battle COVID-19, conformationally selected from many viral capsid structures. Thus, in this project, we will put our nanoplatform in immediate use.

\section{Acknowledgments}

We acknowledge funding support to Professor Holland Cheng by NIH grant numbers: A1095382, EB021230, CA198880, National Institute of Food and Agriculture, as well as the Finland Distinguished Professor Program.

\section{Conflict of Interest}

No conflict of interest.

\section{Bibliography}

1. Zafrullah M., et al. "Acidic pH enhances structure and structural stability of the capsid protein of hepatitis E virus". Biochemical and Biophysical Research Communications 313.1 (2004): 67-73.

2. Kawano M., et al. "Design Platforms of Nanocapsules for Human Therapeutics or Vaccines, in Development of Vaccines". John Wiley and Sons, Inc (2011): 125-139.

3. Xing L.., et al. "Recombinant hepatitis E capsid protein selfassembles into a dual-domain $\mathrm{T}=1$ particle presenting native virus epitopes". Virology 265.1 (1999): 35-45.

4. Chen CC., et al. "Chemically activatable viral capsid functionalized for cancer targeting". Nanomedicine 11.4 (2016): 377 390.

5. Jariyapong P., et al. "Chimeric hepatitis E virus-like particle as a carrier for oral-delivery". Vaccine 31.2 (2013): 417-424.

6. Chen CC., et al. "Tissue targeted nanocapsids for oral insulin delivery via drink". Pharmaceutical Patent Analyst 7.3 (2018): 121-127.

7. Chen CC SM., et al. "Nanoparticle Encapsulating Nano-Thernostic agent as Modularized Capsule". Advanced Research in Gastroenterology and Hepatology 5.5 (2017): 555674.
8. Holla P., et al. "Chapter Sixteen - Toward Mucosal DNA Delivery: Structural Modularity in Vaccine Platform Design, in Micro and Nanotechnology in Vaccine Development, M. Skwarczynski and I. Toth, Editors., William Andrew Publishing (2017): 303-326.

9. Shizuo G Kamita MAB., et al. "A Noninvasive, Orally Stable, Mucosa-Penetrating Polyvalent Vaccine Platform Based on Hepatitis E Virus Nanoparticle". Synthetic Biology - New Interdisciplinary Science (2019).

10. Lu R., et al. "Genomic characterisation and epidemiology of 2019 novel coronavirus: implications for virus origins and receptor binding". Lancet 395.10224 (2020): 565-574.

11. Zhou P., et al. "A pneumonia outbreak associated with a new coronavirus of probable bat origin". Nature 579.7798 (2020): 270-273.

12. Letko M and V Munster. "Functional assessment of cell entry and receptor usage for lineage $\mathrm{B}$ beta-coronaviruses, including 2019-nCoV". Bio Rxiv (2020).

13. Hoffmann M., et al. "The novel coronavirus 2019 (2019-nCoV) uses the SARS-coronavirus receptor ACE2 and the cellular protease TMPRSS2 for entry into target cells". Bio Rxiv (2020).

14. Ahmed SF., et al. "Preliminary Identification of Potential Vaccine Targets for the COVID-19 Coronavirus (SARS-CoV-2) Based on SARS-CoV Immunological Studies". Viruses 12.3 (2020).

15. Liu C., et al. "Research and Development on Therapeutic Agents and Vaccines for COVID-19 and Related Human Coronavirus Diseases". ACS Central Science 6.3 (2020): 315-331.

16. Liu Y., et al. "Elevated plasma levels of selective cytokines in COVID-19 patients reflect viral load and lung injury". National Science Review 7.6 (2020): 1003-1011.

17. Yang ZY., et al. "A DNA vaccine induces SARS coronavirus neutralization and protective immunity in mice". Nature 428.6982 (2004): 561-564.

18. Deming D., et al. "Vaccine efficacy in senescent mice challenged with recombinant SARS-CoV bearing epidemic and zoonotic spike variants". PLOS Medicine 3.12 (2006): e525.

19. Graham RL., et al. "A live, impaired-fidelity coronavirus vaccine protects in an aged, immunocompromised mouse model of lethal disease". Nature Medicine 18.12 (2012): 1820-1826. 
20. Lin Y., et al. "Identification of an epitope of SARS-coronavirus nucleocapsid protein". Cell Research 13.3 (2003): 141-145.

21. Wang J., et al. "Assessment of immunoreactive synthetic peptides from the structural proteins of severe acute respiratory syndrome coronavirus". Clinical Chemistry 49.12 (2003): 1989-1996.

22. Liu X., et al. "Profile of Antibodies to the Nucleocapsid Protein of the Severe Acute Respiratory Syndrome (SARS)-Associated Coronavirus in Probable SARS Patients". Clinical and Diagnostic Laboratory Immunology 11.1 (2004): 227.

23. Tang F., et al. "Lack of peripheral memory B cell responses in recovered patients with severe acute respiratory syndrome: a six-year follow-up study". Journal of Immunology 186.12 (2011): 7264-7268.

24. Peng H., et al. "Long-lived memory T lymphocyte responses against SARS coronavirus nucleocapsid protein in SARS-recovered patients". Virology 351.2 (2006): 466-475.

25. Fan YY., et al. "Characterization of SARS-CoV-specific memory T cells from recovered individuals 4 years after infection". Archives of Virology 154.7 (2009): 1093-1099.

26. Ng OW., et al. "Memory $\mathrm{T}$ cell responses targeting the SARS coronavirus persist up to 11 years post-infection". Vaccine 34.17 (2016): 2008-2014.

27. Liu WJ., et al. "T-cell immunity of SARS-CoV: Implications for vaccine development against MERS-CoV". Antiviral Research 137 (2017): 82-92.

28. Li CK., et al. "T cell responses to whole SARS coronavirus in humans". Journal of Immunology 181.8 (2008): 5490-5500.

29. Channappanavar R, et al. "Virus-specific memory CD8 T cells provide substantial protection from lethal severe acute respiratory syndrome coronavirus infection". Journal of Virology 88.19 (2014): 11034-11044.

30. Tay MZ., et al. "The trinity of COVID-19: immunity, inflammation and intervention". Nature Reviews Immunology 20.6 (2020): 363-374.

31. Chan JFW., et al. "A familial cluster of pneumonia associated with the 2019 novel coronavirus indicating person-toperson transmission: a study of a family cluster". The Lancet 395.10223 (2020): 514-523.

32. Lan J., et al. "Structure of the SARS-CoV-2 spike receptor-binding domain bound to the ACE2 receptor". Nature 581.7807 (2020): 215-220.
33. Xu Z., et al. "Pathological findings of COVID-19 associated with acute respiratory distress syndrome". The Lancet Respiratory Medicine 8.4 (2020): 420-422.

34. Bisht H., et al. "Neutralizing antibody and protective immunity to SARS coronavirus infection of mice induced by a soluble recombinant polypeptide containing an $\mathrm{N}$-terminal segment of the spike glycoprotein". Virology 334.2 (2005): 160-165.

35. Liu C., et al. "Research and Development on Therapeutic Agents and Vaccines for COVID-19 and Related Human Coronavirus Diseases". ACS Central Science 6.3 (2020): 315-331.

36. Brandtzaeg P. "Function of mucosa-associated lymphoid tissue in antibody formation". Immunological Investigations 39.4-5 (2010): 303-355.

37. Yusuf $H$ and V Kett. "Current prospects and future challenges for nasal vaccine delivery". Human Vaccines and Immunotherapeutics 13.1 (2017): 34-45.

38. Fagarasan $\mathrm{S}$ and T Honjo. "Intestinal IgA synthesis: regulation of front-line body defences". Nature Reviews Immunology 3.1 (2003): 63-72.

39. Shakya AK., et al. "Mucosal vaccine delivery: Current state and a pediatric perspective". Journal of Controlled Release 240 (2016): 394-413.

40. Kang SH., et al. "Oral Vaccine Delivery for Intestinal ImmunityBiological Basis, Barriers, Delivery System, and M Cell Targeting". Polymers 10.9 (2018): 948.

41. Kim SH., et al. "Targeted Delivery of VP1 Antigen of Foot-andmouth Disease Virus to M Cells Enhances the Antigen-specific Systemic and Mucosal Immune Response". Immune Network 13.4 (2013): 157-162.

42. Ghosh S., et al. "siRNA could be a potential therapy for COVID-19”. EXCLI Journal 19 (2020): 528-531.

43. Meng B., et al. "Identification of effective siRNA blocking the expression of SARS viral envelope E and RDRP genes". Molecular Biotechnology 33.2 (2006): 141-148.

44. Wu A., et al. "Genome Composition and Divergence of the Novel Coronavirus (2019-nCoV) Originating in China". Cell Host Microbe 27.3 (2020): 325-328.

45. Wu F., et al. "A new coronavirus associated with human respiratory disease in China". Nature 579.7798 (2020): 265-269. 
46. Baker TS and RH Cheng. "A model-based approach for determining orientations of biological macromolecules imaged by cryoelectron microscopy". Journal of Structural Biology 116.1 (1996): 120-130.

47. Li TC., et al. "Essential elements of the capsid protein for selfassembly into empty virus-like particles of hepatitis E virus". Journal of Virology 79.20 (2005): 12999-3006.

48. Xing L., et al. "Structure of hepatitis E virion-sized particle reveals an RNA-dependent viral assembly pathway". Journal of Biological Chemistry 285.43 (2010): 33175-33183.

49. Takamura S., et al. "DNA vaccine-encapsulated virus-like particles derived from an orally transmissible virus stimulate mucosal and systemic immune responses by oral administration". Gene Therapy 11.7 (2004): 628-635.

50. Xing L., et al. "Spatial configuration of hepatitis E virus antigenic domain". Journal of Virology 85.2 (2011): 1117-1124.

51. Donnelly JJ., et al. "DNA vaccines: progress and challenges". Journal of Immunology 175.2 (2005): 633-639.

52. Corr SC., et al. "M-cells: origin, morphology and role in mucosal immunity and microbial pathogenesis". FEMS Immunology and Medical Microbiology 52.1 (2008): 2-12.

53. Kim SH., et al. "The M cell-targeting ligand promotes antigen delivery and induces antigen-specific immune responses in mucosal vaccination". Journal of Immunology 185.10 (2010): 5787-2795.

54. Garinot M., et al. "PEGylated PLGA-based nanoparticles targeting M cells for oral vaccination". The Journal of Controlled Release 120.3 (2007): 195-204.

55. Kim SH., et al. "M cells expressing the complement C5a receptor are efficient targets for mucosal vaccine delivery". European Journal of Immunology 41.11 (2011): 3219-3229.

56. Chen CC., et al. "Surface Functionalization of Hepatitis E Virus Nanoparticles Using Chemical Conjugation Methods". Journal of Visualized Experiments 135 (2018).

57. Abbott TR., et al. "Development of CRISPR as an Antiviral Strategy to Combat SARS-CoV-2 and Influenza". Cell 181.4 (2020): 865-876 e12.

\section{Assets from publication with us}

- Prompt Acknowledgement after receiving the article

- Thorough Double blinded peer review

- Rapid Publication

- Issue of Publication Certificate

- High visibility of your Published work

Website: https://www.actascientific.com/

Submit Article: https://www.actascientific.com/submission.php Email us: editor@actascientific.com

Contact us: +919182824667 